\section{OPEN ACCESS}

Edited by: Domenico Tamburrino,

San Raffaele Hospital (IRCCS), Italy

Reviewed by:

$\mathrm{Min} \mathrm{Li}$

Sun Yat-sen University Cancer Center

(SYSUCC), China

Giampaolo Perri,

University of Texas MD Anderson

Cancer Center, United States Adam Frampton,

University of Surrey, United Kingdom

*Correspondence: Keith J. Roberts

Keith.Roberts@uhb.nhs.uk

Georgios Gemenetzis georgios.gemenetzis@glasgow.ac.uk

Specialty section: This article was submitted to

Surgical Oncology,

a section of the journal

Frontiers in Oncology

Received: 11 October 2021 Accepted: 01 December 2021 Published: 23 December 2021

Citation:

Gemenetzis G, McKay S, Pathak S, Moir J, Laing $R$, Jamieson NB,

Young AL, Chatzizacharias NA, Giovinazzo F and Roberts KJ (2021)

Surgical Management of Non-

Metastatic Pancreatic Cancer in the

United Kingdom: Results of a Nationwide Survey on Current Practice.

Front. Oncol. 11:791946.

doi: 10.3389/fonc.2021.791946

\title{
Surgical Management of Non- Metastatic Pancreatic Cancer in the United Kingdom: Results of a Nationwide Survey on Current Practice
}

Georgios Gemenetzis ${ }^{1 *}$, Siobhan McKay ${ }^{2}$, Samir Pathak ${ }^{3}$, John Moir ${ }^{4}$, Richard Laing ${ }^{2}$, Nigel B. Jamieson ${ }^{5}$, Alastair L. Young ${ }^{6}$, Nikolaos A. Chatzizacharias ${ }^{2}$, Francesco Giovinazzo ${ }^{7}$ and Keith J. Roberts ${ }^{2 *}$

${ }^{1}$ Department of Hepatobiliary (HPB) and Transplant Surgery, Royal Infirmary Edinburgh, Edinburgh, United Kingdom, ${ }^{2}$ Liver Unit, Queen Elizabeth University Hospital Birmingham, Birmingham, United Kingdom, ${ }^{3}$ Department of Surgery, University Hospitals Bristol NHS Foundation Trust, Bristol, United Kingdom, ${ }^{4}$ Department of Hepatobiliary, Pancreatic and Transplant Surgery, The Freeman Hospital, Newcastle, United Kingdom, ${ }^{5}$ West of Scotland Pancreatic Unit, Glasgow Royal Infirmary, Glasgow, United Kingdom, ${ }^{6}$ Department of Pancreatic Surgery, St James's University Hospital, Leeds, United Kingdom, ${ }^{7}$ Department of General Surgery and Liver Transplantation, Fondazione Policlinico Universitario Agostino Gemelli IRCCS, Roma, Italy

Background: It is presently unclear what clinical pathways are followed for patients with non-metastatic PDAC in specialised centres for pancreatic surgery across the United Kingdom (UK).

Methods: Between August 2019 and August 2020 an electronic survey was conducted aiming at a national cohort of pancreatic surgeons in the UK. Participants replied to a list of standardised questions and clinical vignettes, and data were collected and analysed focusing on management preferences, resectability criteria, and contraindications to surgery.

Results: Within the study period, 65 pancreatic surgeons from 27 specialist centres in the UK (96\%) completed the survey. Multidisciplinary team meetings are utilised universally for the management of patients with PDAC, however, different staging systems for resectability classification are being applied. In borderline resectable PDAC, most surgeons were keen to proceed with surgical exploration post NAT, but differences were noted in preferred chemotherapy regimens. Surgeons from standard volume institutions performed fewer vein resections annually and were more likely to deem patients with locally advanced PDAC as unresectable. Intra-institutional variability in patient management was also present and ranging between $20-80 \%$.

Conclusions: Significant variability in the surgical management of non-metastatic PDAC was identified both on inter- and intra-institutional level.

Keywords: pancreatic cancer, survey, neoadjuvant, surgery, practice 


\section{INTRODUCTION}

Pancreatic adenocarcinoma (PDAC) remains a devastating disease with an extremely poor prognosis (1). The 5-year overall survival (OS) rate in the United Kingdom (UK) is approximately $7 \%$ and has not improved significantly in the past 30 years (2). Furthermore, there is a $17 \%$ increase in the incidence of PDAC cases at the same time period (3) resulting to more than 10,000 new diagnoses annually in the UK. In patients with non-metastatic PDAC, margin-negative (R0) surgical resection of the primary tumour is the primary therapeutic approach to achieve long-term survival $(4,5)$. Advancements in surgical quality and perioperative care and the introduction of more efficient perioperative systemic treatment have further improved patient outcomes $(6,7)$.

The paradigm of surgical management is shifting in patients with borderline resectable (BR) and locally advanced (LA) PDAC. Several classification systems in the literature define BR and LAPDAC (8-10) focusing on variable degrees of vessel involvement by the tumour; yet the presence of different staging systems results in evolving definitions and a nuanced distinction between them (11). In BR-PDAC, the introduction of neoadjuvant/induction systemic treatment (NAT) was based on its potential advantages: addressing occult micrometastatic disease in the preoperative setting, avoiding unnecessary surgery in tumours with aggressive biology, increasing the likelihood of R0 resection, and improving delivery rates of systemic treatment. Recent prospective trials have demonstrated a potential benefit in overall survival in patients with BR-PDAC who underwent NAT $(12,13)$, and emerging data suggest potential future practice changes.

In the UK, the National Institute for Health and Care Excellence (NICE) pancreatic cancer guidelines recommend surgery for resectable cancer and advise the utilisation of NAT in resectable and BR-PDAC only as part of clinical trials (14). However, trial availability varies over time and patient recruitment can often be challenging. Therefore, it appears to be presently unclear what treatment pathways are chosen by the various specialist pancreatic surgical teams within the UK. A previous prospective audit on diagnostic pathways in PDAC (RICOCHET) demonstrated wide variations in practice and indicated the need for clinical pathway optimisation (15). The present study aims to further characterize variations in surgical management of patients with non-metastatic PDAC in specialised centres across the UK, assess and investigate differences in practice, and determine potential parameters for pathway standardisation.

\section{METHODS}

\section{Study Design and Participants}

This study is a nationwide electronic survey that was carried out between August 2019 and August 2020. The survey was constructed on a web-based platform [REDCap, Vanderbilt University, TN, USA (16)] and was distributed via email, following established guidelines on electronic surveys (17). It was designed to target all consultant surgeons who hold a permanent or locum post and perform pancreatic surgery in institutions within the National Health System (NHS) across the UK. Identification of potential participants was performed through direct communications with every specialist unit in the UK. Senior trainees and fellows were excluded from this study. Eligible surgeons received an official email with the survey details and reminder emails were sent to participants who did not respond during the course of the study. Participants were informed in advance that the survey would require approximately 10-15 minutes to complete and that they needed to provide personal identification details including name, email address, and hospital. Additional information was asked to be provided regarding the annual volume of pancreatic resections performed in the institution of each participant. No rewards were offered for participation in the survey.

\section{Survey Contents: Questions and Clinical Vignettes}

The survey was designed to assess surgical practice in PDAC focusing mainly on anatomical considerations, surgical techniques, and application of perioperative systemic treatment. An additional focus of the study was to acquire information regarding the organisation of institutional multidisciplinary team (MDT) meetings and structure of patient pathways. The rationale was to explore in detail differences in individual practices regarding the management of patients with non-metastatic PDAC. Therefore, the survey questionnaire was divided into two sections: the first included questions regarding practice characteristics and surgical decision-making, and the second comprised of five clinical vignettes. Most of the individual questions allowed one answer, however, select ones prompted participants to check multiple answers that applied.

The vignettes corresponded to five clinical scenarios that were created based on real patients (Supplementary File 1). Four vignettes included cases that covered the range of non-metastatic PDAC management (resectable, borderline resectable, and locally advanced tumours) and the fifth vignette addressed the management of a patient with a resectable pancreatic head primary and an indeterminate lung lesion. The vignette description contained all pertinent information to the participants, including patient demographics, clinical details, and anatomical characteristics of the tumour - de-identified CT images of real patients were utilised in three scenarios for clarity. In vignettes with BR-PDAC and LA-PDAC, all patients had previously received NAT with variable degrees of radiological and biomarker response. Three of the presented cases included follow-up questions to better assess surgical rationale. The full survey is available in the appendix (Supplementary File 1).

\section{Statistical Analysis}

For optimal identification of surgical management, participants were stratified in three tiers, based on the volume of pancreatic resections in their institutions. Since all participants are practising 
in dedicated hepatopancreatobiliary centres identified as highvolume in the literature [ $>20$ pancreatectomies annually (18)], the stratification occurred as follows: standard volume $(<50$ pancreatectomies/year), high volume (50-100 pancreatectomies/ year), and very high volume (>100 pancreatectomies/year).

All participant data were de-identified prior to final analysis. Categorical and non-parametric data are presented as numbers and percentages. Continuous variables were compared with the Mann-Whitney $U$ test and categorical variables with the $\chi^{2}$ test. Intra-institutional variability in replies was calculated using Krippendorf's $\alpha$ co-efficient on nominal data, and results were interpreted and presented as percentages (0-100\%). For all tests, statistical significance was accepted with a two-sided $p$ value of $<0.05$. The SPSS statistical software version 25.0 (SPSS Inc., Chicago, IL) was utilised for statistical analysis.

\section{RESULTS}

Within the study period, approximately 120 individual emails were sent to candidate participants, excluding reminder communications. In total, 65 eligible participants completed the survey, accounting approximately for $58 \%$ of all estimated HPB consultants in the UK and representing 27 of the 28 national referral centres. The median completion rate of the survey was $94.6 \%$ and all responses were included in the final analysis. The majority of the participants practise in academic institutions $(n=55)$ and 21 surgeons are also performing liver and/or pancreas transplantation. Regarding geographical distribution, $72 \%$ of the responders were based in England $(n=47), 23 \%$ in Scotland $(n=15), 3 \%$ in Wales $(n=2)$, and $2 \%$ in Northern Ireland $(n=1)$. According to the predetermined volume-based stratification, 14 surgeons practise in standardvolume $(22 \%), 20$ in high-volume $(31 \%)$, and 31 in very high-volume institutions (48\%).

All participants stated that MDT meetings are conducted in their respective institutions for evaluation and management of PDAC patients (Table 1). Most MDT meetings utilise the National Comprehensive Cancer Network (NCCN) guidelines for assessment of tumour resectability (19) (69\%); surgeons from very high-volume centres reported increased adherence to the NCCN guidelines compared to standard-volume institutions

TABLE 1 | Surgeon preferences for management of non-metastatic pancreatic cancer stratified by annual institutional volume of pancreatic resections.

\begin{tabular}{|c|c|c|c|c|c|}
\hline Management Preferences & $\begin{array}{c}\text { All } \\
(n=65)\end{array}$ & $\begin{array}{l}\text { Standard volume } \\
\qquad(n=14)\end{array}$ & $\begin{array}{l}\text { High volume } \\
\quad(n=20)\end{array}$ & $\begin{array}{l}\text { Very high volume } \\
\qquad(\mathrm{n}=31)\end{array}$ & p-value \\
\hline NAT in resectable PDAC, $n(\%)$ & & & & & 0.106 \\
\hline Selectively & $20(31 \%)$ & $2(14 \%)$ & $6(30 \%)$ & $12(39 \%)$ & \\
\hline Routinely & $3(4 \%)$ & $0(0 \%)$ & $3(15 \%)$ & $0(0 \%)$ & \\
\hline \multicolumn{6}{|l|}{ Rationale for NAT in resectable PDAC, $n$ (\%) } \\
\hline Clinical trial & $3(5 \%)$ & $1(7 \%)$ & $0(0 \%)$ & $2(6 \%)$ & 0.191 \\
\hline Preoperative pancreatitis & $1(2 \%)$ & $0(0 \%)$ & $0(0 \%)$ & $1(3 \%)$ & \\
\hline Vessel contact & $4(6 \%)$ & $0(0 \%)$ & $1(5 \%)$ & $3(10 \%)$ & \\
\hline Offer resection in resectable patients with high CA $19-9, n$ (\%) & $56(86 \%)$ & $13(93 \%)$ & $16(80 \%)$ & 27 (87\%) & 0.289 \\
\hline \multicolumn{6}{|l|}{ CA 19-9 cut-off for NAT in resectable patients, $(\mathrm{U} / \mathrm{ml})^{\mathrm{a}}$} \\
\hline$<250$ & $5(8 \%)$ & $2(14 \%)$ & $1(5 \%)$ & $2(6 \%)$ & 0.415 \\
\hline $250-500$ & $12(18 \%)$ & $2(14 \%)$ & $4(20 \%)$ & $6(19 \%)$ & \\
\hline Alliance & $3(5 \%)$ & $1(7 \%)$ & $1(5 \%)$ & $1(3 \%)$ & \\
\hline MD Anderson & $4(6 \%)$ & $2(14 \%)$ & $1(5 \%)$ & $1(3 \%)$ & \\
\hline Other & $2(3 \%)$ & $0(0 \%)$ & $1(5 \%)$ & $1(3 \%)$ & \\
\hline None & $9(14 \%)$ & $4(29 \%)$ & $3(15 \%)$ & $2(6 \%)$ & \\
\hline Multiple & $2(3 \%)$ & $0(0 \%)$ & $1(5 \%)$ & $1(3 \%)$ & \\
\hline \multicolumn{6}{|l|}{ Universal resectability classification system, $n$ (\%) } \\
\hline Useful/very useful & $40(62 \%)$ & $8(57 \%)$ & $13(65 \%)$ & $19(61 \%)$ & 0.654 \\
\hline Not useful & 25 (38\%) & $6(43 \%)$ & $7(35 \%)$ & $12(39 \%)$ & \\
\hline MDT report format, $n(\%)$ & & & & & 0.565 \\
\hline Free text description & $63(97 \%)$ & $13(93 \%)$ & $20(100 \%)$ & $30(97 \%)$ & \\
\hline Template with options & $2(3 \%)$ & $1(7 \%)$ & $0(0 \%)$ & $1(3 \%)$ & \\
\hline \multicolumn{6}{|l|}{ Standardisation of MDT reporting, $n$ (\%) } \\
\hline Definitely/Yes & $33(51 \%)$ & $8(57 \%)$ & $8(40 \%)$ & $17(55 \%)$ & 0.339 \\
\hline
\end{tabular}

NAT, neoadjuvant treatment; PDAC, pancreatic adenocarcinoma; CA19-9, carcinoembryonic antigen; MDT, multidisciplinary tumour board; NCCN, National Comprehensive Cancer Network; ${ }^{\text {a }}$ issing values $n=10$ (15\%). P-values in bold are statistically significant. 
( $p=0.036$ ). Interestingly, $14 \%$ of participants replied that they do not utilise a dedicated resectability classification system. Additionally, in the vast majority of cases MDT reports are presented as free text and only 3\% of participants mentioned outcome reporting in a pre-drafted template. Most surgeons find useful the application of a universal resectability classification system nationally (62\%) and approximately half of them support the standardisation of MDT reporting across the UK.

Surgeon preferences on the management of non-metastatic PDAC are available in Tables $\mathbf{1}, \mathbf{2}$. For upfront resectable PDAC, $65 \%$ of participants would never offer NAT in patients with performance status of $0-1$, even in the presence of high CA19-9 levels (86\%). Surgeons who supported the utilisation of NAT in resectable PDAC would offer it in patients with increased risk of positive margin (R1) resection due to contact of the tumour with the portal/superior mesenteric complex $(n=13)$, or within a clinical trial $(n=3)$.

Participants practising in very high-volume institutions for pancreatic surgery performed statistically significantly more vascular resections in pancreatectomies compared to ones in standard or high-volume centres $(p<0.001)$. In BR and LAPDAC, no differences were identified between surgeons regarding preferred technique for vascular reconstruction. Similarly, the importance of radiological involvement of regional vascular structures by the tumour was equally distributed: in veins length of involved segment and presence of cavernous transformation, and in arteries degree of circumferential involvement and presence of narrowing appear to be more significant in preoperative assessment (Table 2). Figure 1 demonstrates the distribution of upfront surgical

TABLE 2 | Technical surgical considerations in the management of borderline resectable and locally advanced pancreatic adenocarcinoma

\begin{tabular}{|c|c|c|c|c|c|}
\hline Management Preferences & All $(n=65)$ & Standard volume $(n=14)$ & High volume $(n=20)$ & Very high volume $(n=31)$ & p-value \\
\hline Vascular resections in past 2 years, $n(\%)^{a}$ & & & & & $<0.001$ \\
\hline $0-1$ & $3(5 \%)$ & $0(0 \%)$ & $0(0 \%)$ & $3(10 \%)$ & \\
\hline $2-5$ & $29(45 \%)$ & $12(86 \%)$ & $7(35 \%)$ & $10(32 \%)$ & \\
\hline $6-10$ & $20(31 \%)$ & $1(7 \%)$ & $9(45 \%)$ & $10(32 \%)$ & \\
\hline$>10$ & $12(18 \%)$ & $1(7 \%)$ & $3(15 \%)$ & $8(26 \%)$ & \\
\hline Preferred technique for vascular reconstruction, $n(\%)$ & & & & & 0.286 \\
\hline Venorrhaphy only & $3(5 \%)$ & $1(7 \%)$ & $1(5 \%)$ & $2(6 \%)$ & \\
\hline End-to-end anastomosis only & $9(14 \%)$ & $0(0 \%)$ & $0(0 \%)$ & $4(13 \%)$ & \\
\hline Interposition graft only & $0(0 \%)$ & $0(0 \%)$ & $0(0 \%)$ & $0(0 \%)$ & \\
\hline Multiple based on individual patient & $54(83 \%)$ & $11(79 \%)$ & 18 (90\%) & $25(81 \%)$ & \\
\hline Vein characteristics, $n(\%)^{\mathrm{b}}$ & & & & & 0.693 \\
\hline Length of involvement & & & & & \\
\hline Important/very important & $54(83 \%)$ & $13(93 \%)$ & 17 (85\%) & $24(77 \%)$ & \\
\hline Somewhat important & $3(5 \%)$ & $0(0 \%)$ & $1(5 \%)$ & 2 (6\%) & \\
\hline Not important & $3(5 \%)$ & $1(7 \%)$ & $1(5 \%)$ & $1(3 \%)$ & \\
\hline Degree of circumferential involvement & & & & & 0.647 \\
\hline Important/very important & $48(74 \%)$ & $11(79 \%)$ & $14(70 \%)$ & $23(74 \%)$ & \\
\hline Somewhat important & $10(15 \%)$ & $2(14 \%)$ & $3(15 \%)$ & $5(16 \%)$ & \\
\hline Not important & $2(3 \%)$ & $0(0 \%)$ & $1(5 \%)$ & $1(3 \%)$ & \\
\hline Presence of narrowing & & & & & 0.161 \\
\hline Important/very important & $41(63 \%)$ & $11(79 \%)$ & $9(45 \%)$ & $21(68 \%)$ & \\
\hline Somewhat important & $15(23 \%)$ & $2(14 \%)$ & $6(30 \%)$ & 7 (23\%) & \\
\hline Not important & 5 (8\%) & $0(0 \%)$ & $4(20 \%)$ & $1(3 \%)$ & \\
\hline Cavernous transformation & & & & & 0.326 \\
\hline Important/very important & 59 (91\%) & $14(100 \%)$ & $18(90 \%)$ & $27(87 \%)$ & \\
\hline Somewhat important & $1(2 \%)$ & $0(0 \%)$ & $0(0 \%)$ & 1 (3\%) & \\
\hline Not important & $1(2 \%)$ & $0(0 \%)$ & $0(0 \%)$ & $1(3 \%)$ & \\
\hline $1^{\text {st }}$ jejunal branch involvement & & & & & 0.105 \\
\hline Important/very important & $53(82 \%)$ & $14(100 \%)$ & $14(70 \%)$ & 25 (81\%) & \\
\hline Somewhat important & 6 (9\%) & $0(0 \%)$ & $2(10 \%)$ & $4(13 \%)$ & \\
\hline Not important & $3(5 \%)$ & $0(0 \%)$ & $1(5 \%)$ & $2(6 \%)$ & \\
\hline Artery characteristics, $n(\%)^{\mathrm{c}}$ & & & & & 0.628 \\
\hline \multicolumn{6}{|l|}{ Length of involvement } \\
\hline Important/very important & 57 (88\%) & 12 (86\%) & $18(90 \%)$ & 27 (87\%) & \\
\hline Somewhat important & 2 (3\%) & 1 (7\%) & $0(0 \%)$ & 1 (3\%) & \\
\hline Not important & $5(8 \%)$ & $0(0 \%)$ & $2(10 \%)$ & $3(10 \%)$ & \\
\hline Degree of circumferential involvement & & & & & 0.342 \\
\hline Important/very important & $62(95 \%)$ & $14(100 \%)$ & 19 (95\%) & $29(94 \%)$ & \\
\hline Somewhat important & $1(2 \%)$ & $0(0 \%)$ & $1(5 \%)$ & $0(0 \%)$ & \\
\hline Not important & $2(3 \%)$ & $0(0 \%)$ & $0(0 \%)$ & $2(6 \%)$ & \\
\hline Presence of narrowing & & & & & 0.531 \\
\hline Important/very important & 54 (83\%) & $12(86 \%)$ & $18(90 \%)$ & $24(77 \%)$ & \\
\hline Somewhat important & $4(6 \%)$ & $2(14 \%)$ & $0(0 \%)$ & $2(6 \%)$ & \\
\hline Not important & $7(11 \%)$ & $0(0 \%)$ & $2(10 \%)$ & $5(16 \%)$ & \\
\hline
\end{tabular}

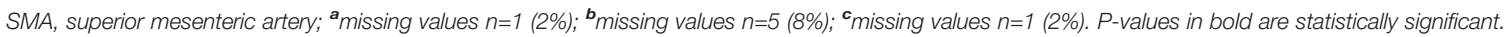




\section{Upfront Surgical Resection}

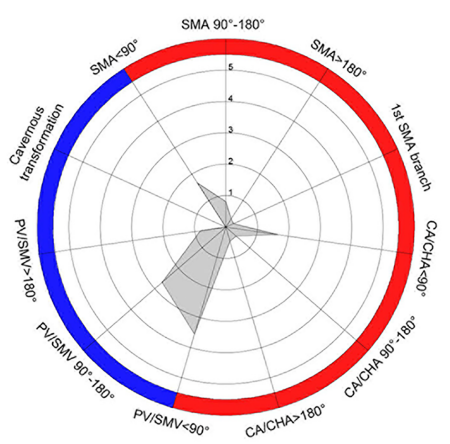

Standard Volume

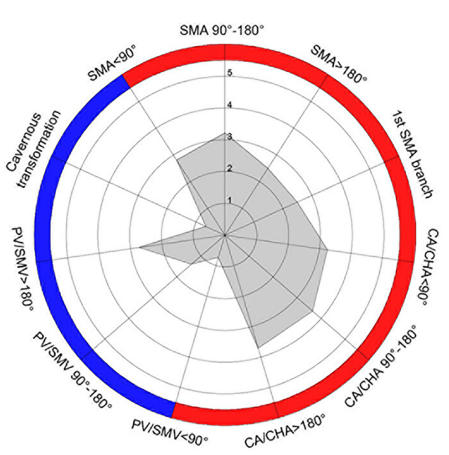

Standard Volume

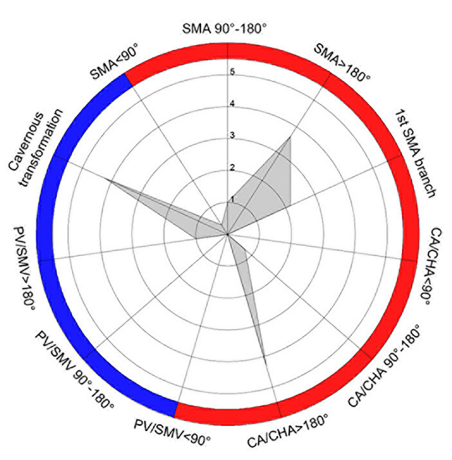

Standard Volume

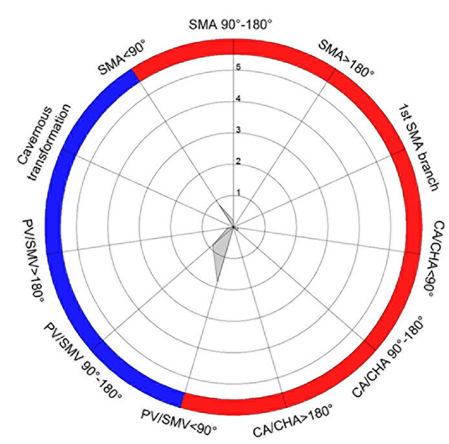

High Volume

Neoadjuvant Therapy

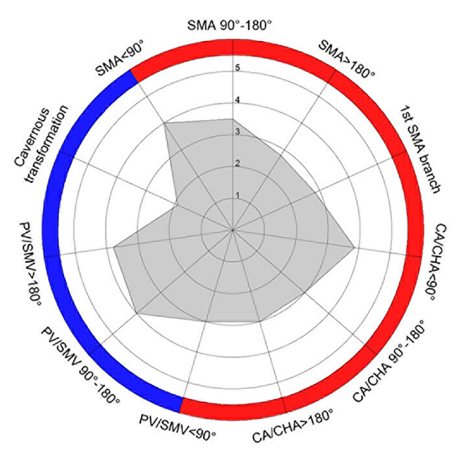

High Volume

Unresectable disease/ palliative care

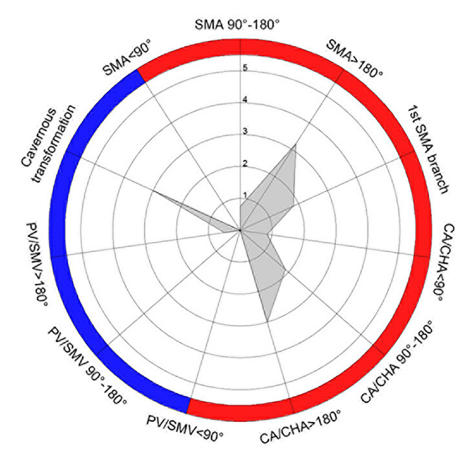

High Volume

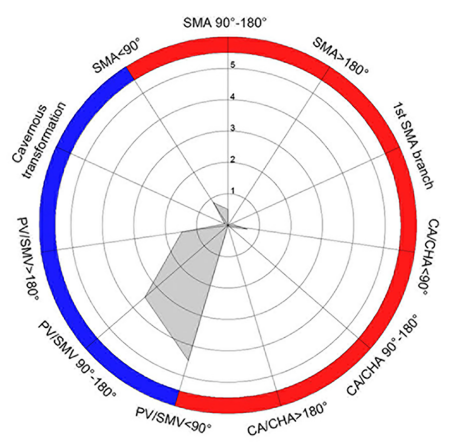

Very High Volume

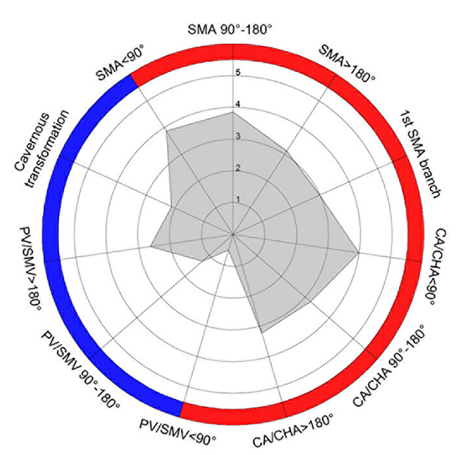

Very High Volume

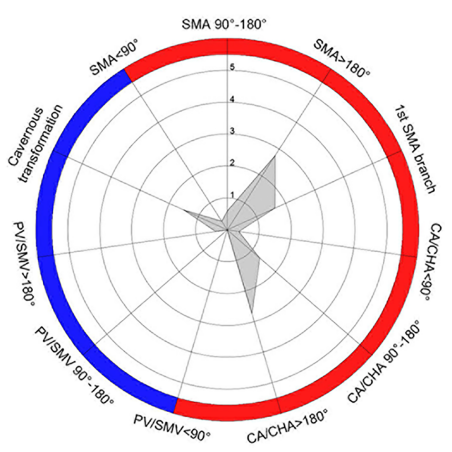

Very High Volume

FIGURE 1 | Radar chart depiction of upfront surgical resection, utilisation of neoadjuvant treatment, and declaration of unresectability in initial PDAC diagnosis based on variable vessel involvement by the primary tumour between the three volume-related tiers. Blue-coloured and red-coloured areas indicate different degrees of venous and arterial involvement, respectively; $1-5$ enumeration refers to percentage of responses $(1=20 \%, 5=100 \%)$. 
resection, NAT, and declaration of unresectability based on different degrees of venous and arterial involvement between participants in the three volume-based tiers (Figure 1; baseline histogram available in Supplementary File 2). Surgeons in highvolume centres were less likely to offer upfront resection in patients with tumours with any degree of vessel involvement compared to surgeons in the other two tiers. Additionally, surgeons in standard-volume institutions offered NAT in fewer cases and declared more patients unresectable, especially in the presence of cavernous transformation or encasement of the superior mesenteric artery $\left(>180^{\circ}\right.$ involvement). Despite management variations, statistically significant differences were not identified between the three tiers.

\section{Clinical Vignettes}

The response distribution for the clinical vignettes is available in Figure 2. The majority of surgeons would offer surgical resection in a patient with resectable PDAC and high CA19-9, and surgical exploration in a patient with BR-PDAC and stable disease on CT after four months of gemcitabine/nab-paclitaxel $(n=60$, in both cases). In the latter case, the rationale for continuation of NAT $(n=5)$ and re-evaluation was mainly concern about R0 margin resection and uncertainty regarding tumour biology; all surgeons who advocated for NAT practised in high or very high-volume institutions and most preferred additional systemic treatment with FOLFIRINOX $(n=3)$.

The third clinical vignette presented a fit patient with a pancreatic body PDAC and involvement of the coeliac artery, who is a potential candidate for distal pancreatectomy with coeliac artery resection (DP-CAR). Most participants opted for NAT and surgical exploration after stable disease/no progression or treatment response on CT per RESIST 1.1 criteria (20) (38\% and $20 \%$, respectively). Interestingly, $38 \%$ of participants $(n=25$, similarly distributed between the three tiers) replied that they would refer the patient for palliative systemic treatment without an option for surgical exploration. In a similar scenario of a young patient with LA-PDAC, half of surgeons $(n=32)$ would offer surgical exploration after approximately one year of systemic treatment without disease progression; $78 \%$ of them $(n=25)$ would declare the primary tumour unresectable if arterial reconstruction were deemed necessary intraoperatively. The final vignette described the likely scenario of a patient with a BRPDAC and an indeterminate lung nodule on imaging: 15\% of participants would not proceed with exploration after four months of NAT and would require biopsy of the lung lesion or continuation of systemic treatment.

\section{Intra-Institutional Variation}

Review and analysis of responses from surgeons in the same institution demonstrated significant variation in practice (Figure 3). This was reflected in both the individual questions and the clinical vignettes, where $20-80 \%$ of responses were different within the same centre. Convergence in decisionmaking and surgical practice was not observed in any of the three tiers. Complete agreement in clinical vignette responses was observed only from surgeons in one institution in the standard volume and one in the very-high volume tier $(25 \%$ and $12.5 \%$, respectively).

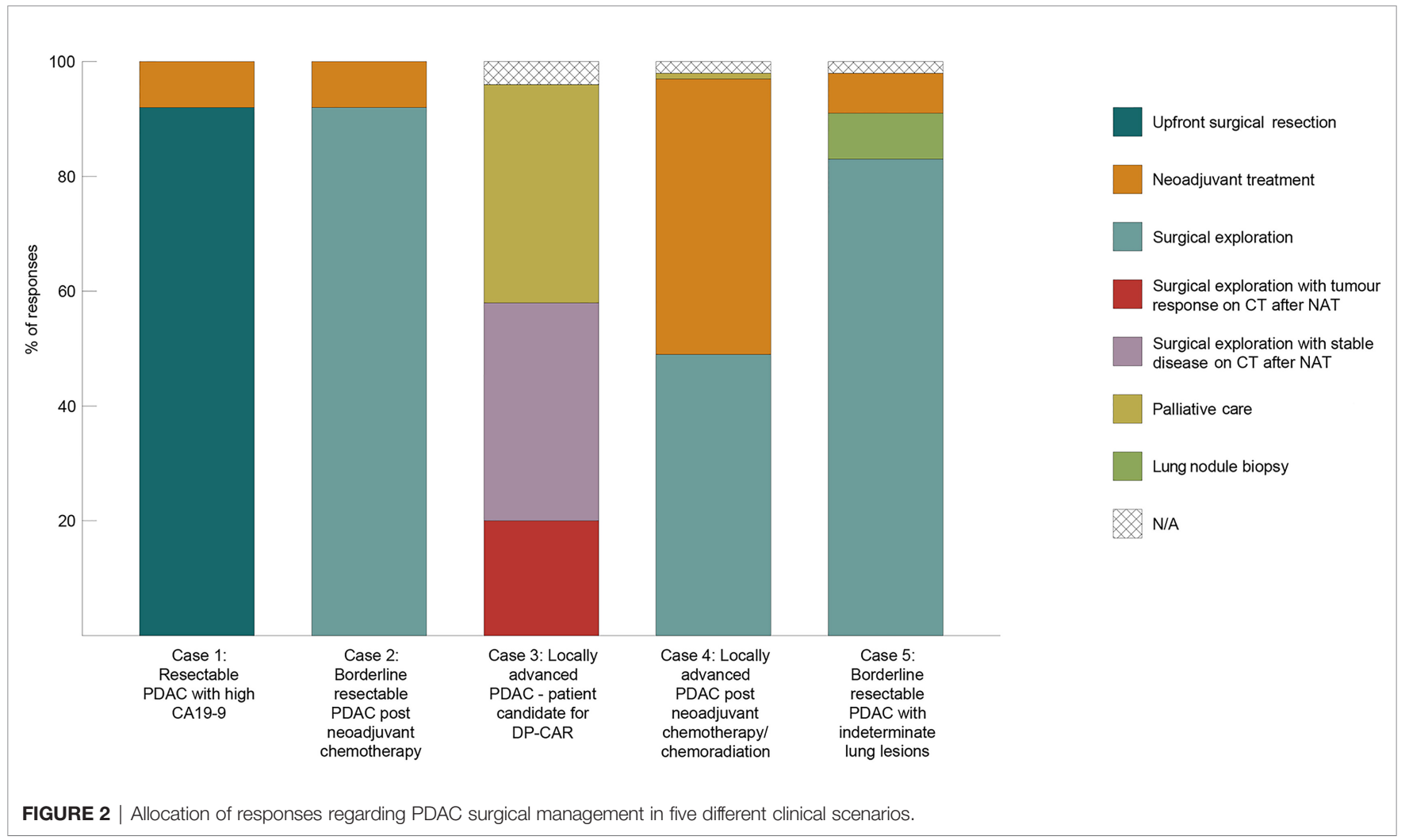


$\%$

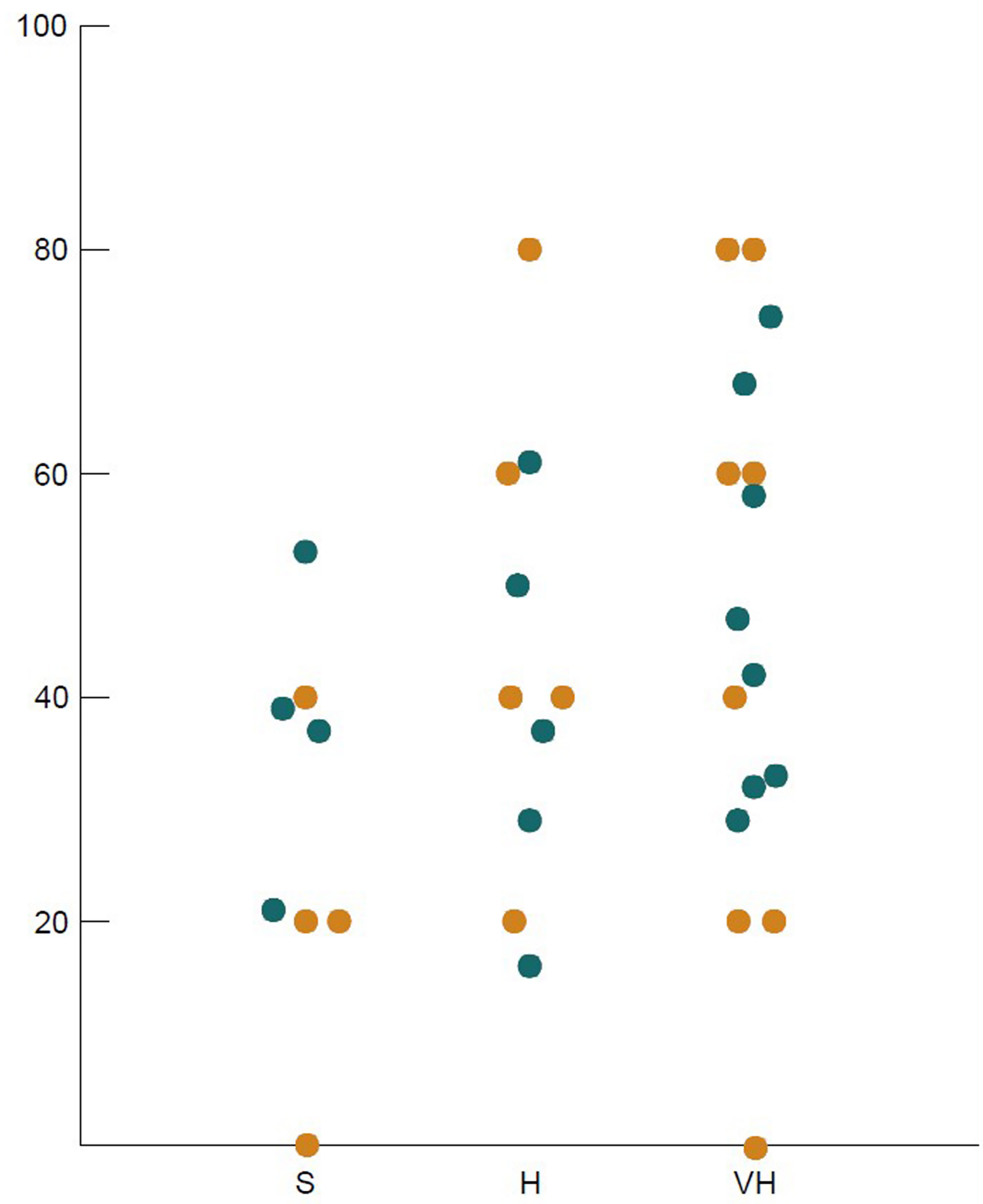

FIGURE 3 | Intra-institutional variation in responses: each dot represents the percentage of questions across the survey with different answers from surgeons in the same institution. Columns refer to standard $(\mathrm{S})$, high $(\mathrm{H})$, and very high $(\mathrm{VH})$ volume hospitals; orange dots: clinical vignettes, blue dots: other questions.

\section{DISCUSSION}

The rationale for this study was to assess and define variations in surgical practice and to our knowledge is the first to provide an insight into the landscape of current surgical management of non-metastatic PDAC in the UK. The replies collected from this survey represent the majority of surgeons who perform pancreatic resections nationally and demonstrate significant 
variability in therapeutic approach and decision-making, both in inter-, and intra-institutional level. These differences were more prominent in the management of BR-PDAC and LA-PDAC, primarily in terms of anatomic criteria for resectability of the primary tumour.

The highest rate of agreement among the responders was observed in the management of patients with resectable PDAC; approximately $90 \%$ advocate for upfront surgery and adjuvant systemic treatment. Traditionally, surgical resection of primary pancreatic tumours without vessel involvement is the treatment of choice in eligible patients (21) and is recommended by national and international guidelines (22). Moreover, "fasttrack" clinical pathways can facilitate prompt patient access to surgery and result in higher resection rates and improved outcomes (23). Although postoperative complications can delay the administration of systemic treatment $(24,25)$, recent advancements in chemotherapy $(6,7)$ have had a significant impact in recurrence-free and overall survival rates (26).

However in this survey, treatment of patients with resectable PDAC was not significantly affected by high CA19-9 levels or poor patient performance status (PS) (27). More specifically, $86 \%$ and $51 \%$ of surgeons would offer upfront resection in patients with CA19-9 $>1000 \mathrm{U} / \mathrm{ml}$ or PS $\geq 2$, respectively. CA19-9 is currently the only available biomarker in clinical practice for PDAC and despite mediocre sensitivity and specificity, high values are suggestive of aggressive tumour biology (28) and have been associated with early disease recurrence (29). The study of biological behaviour in PDAC gradually introduced further criteria for resectability beyond anatomic considerations $(11,30)$ aiming at the improvement of recurrence rates and overall survival. Since optimal outcomes in PDAC are observed when a combination of local and systemic treatment is provided, the concept of NAT in resectable PDAC was developed with two objectives in mind: 1 . test tumour biology and better select patients who are eligible for surgery, and 2 . increase the percentage of patients who received chemotherapy. Retrospective studies on NAT in resectable PDAC have demonstrated increased rates in delivery of intended systemic therapy (31), and the recent SWOG S1505 trial showed that this is a feasible approach that needs to be investigated further (32). In this study, less than $10 \%$ of participants from high and very high-volume institutions would routinely offer this approach and only within a clinical trial, as suggested by the NICE guidelines.

Limited variation was identified in the management of BRPDAC, since the majority of surgeons support utilisation of NAT and surgical exploration. The concept of preoperative chemotherapy and chemoradiation in BR-PDAC was developed under the prism of vessel involvement by the tumour and increased probability of $\mathrm{R} 1$ resection with a subsequent effect on patient survival $(4,33)$. Even though the percentage of patients who eventually undergo surgical resection may be lower, the recent prospective PREOPANC and ESPAC$5 \mathrm{~F}$ trials demonstrated improved tumour response and a survival benefit with NAT and surgery $(12,13)$, indicating a paradigm shift in the surgical management of these patients.
Advancements in surgical techniques, perioperative care, and systemic treatment options have significantly improved outcomes in patients with LA-PDAC. Recent retrospective series have demonstrated that surgical resection in LA-PDAC post induction therapy is feasible (34-36) and has a direct impact on patient survival $(37,38)$. However, most of the current practice is based on observational data and therefore, the lack of consensus in LA-PDAC management shown in this study is not surprising. Participants expressed different opinions regarding time and regimen of induction therapy, most likely based on single-centre retrospective data. These results coincide with differing views regarding number of chemotherapy cycles, or any survival benefit in utilisation of radiation therapy in the preoperative setting $(39,40)$.

Since tumour response to induction therapy in LA-PDAC is evaluated almost exclusively based on imaging, anatomic criteria are primarily utilised by surgeons to assess resectability and reconstructability. This combination of subjective radiological evaluation and low specificity and sensitivity of imaging modalities (41) can explain the limited role of surgery in LAPDAC demonstrated in this study. The role of CT/PET has been previously investigated regarding assessment of resectability and has proven to provide an advantage in identifying active disease versus fibrosis, especially after neoadjuvant treatment (42). Another important factor appears to be the limited exposure to complex vascular reconstruction: participants from standardvolume hospitals performed significantly smaller number of vein resections and subsequently were more likely to declare a locally advanced tumour as unresectable, and vice versa. However, almost all surgeons in this study would declare a tumour unresectable intraoperatively if arterial involvement were identified, particularly of the superior mesenteric artery. Arterial resection and reconstruction in PDAC have been previously reported in very selective cases (43), but is characterised by high rates of postoperative complications and questionable survival benefit (44).

The variability in the assessment of patients with PDAC shown in this study indicates that the decision-making process is a combination of guideline utilisation, surgical skills, and individual experience within an MDT setting. Previous studies have reported differences in MDT outcomes between institutions and deviations in assessment of resectability or tumour response (45-47), extending beyond classification systems. This study further underlines that these differences are essentially present on an intra-institutional level and illustrates the necessity for standardisation of MDT reporting, which is also supported by the majority of participants.

This study has several important limitations. Firstly, even though response rate was $>50 \%$, the sample size was relatively small ( $n=65)$ making identification of statistically significant differences problematic. Additionally, the survey was eponymous and therefore research bias that may have affected the responses (Hawthorne effect) was introduced. Limited information regarding previous exposure of participating surgeons to the management of BR-DPAC and LA-PDAC post 
neoadjuvant therapy were also available. Most importantly, it remains unclear if the demonstrated differences in management translate into differences in patient outcomes, since they were not the scope of this study. Lastly, this study was primarily performed before the ongoing COVID-19 pandemic, which has significantly affected the management of PDAC patients and is expected to have significant effect on resection rates and survival $(48,49)$.

Following previous reports (15), the present study shows wide variations in the surgical management of non-metastatic PDAC across the UK. Even though pancreatic surgery is centralised in high-volume centres, a need for creation of a nationwide patient outcome database is underlined. This will allow identification and standardisation of optimal clinical pathways via audit processes within the NHS, similar to the National Surgical Quality Improvement Program in the United States and the British Transplantation Society in the United Kingdom, aiming primarily at the complex management of patients with $\mathrm{BR}$ and LA-PDAC.

\section{REFERENCES}

1. Rahib L, Smith BD, Aizenberg R, Rosenzweig AB, Fleshman JM, Matrisian LM. Projecting Cancer Incidence and Deaths to 2030: The Unexpected Burden of Thyroid, Liver, and Pancreas Cancers in the United States. Cancer Res (2014) 74(11):2913-21. doi: 10.1158/0008-5472.CAN-14-0155

2. Pancreatic Cancer Statistics 2015 . Available at: https://www.cancerresearchuk. org/health-professional/cancer-statistics/statistics-by-cancer-type/ pancreatic-cancer.

3. Blackford AL, Canto MI, Klein AP, Klein AP, Hruban RH, Goggins M. Recent Trends in the Incidence and Survival of Stage 1a Pancreatic Cancer: A Surveillance, Epidemiology, and End Results Analysis. J Natl Cancer Inst (2020) 112(11):1162-9. doi: 10.1093/jnci/djaa004

4. Ghaneh P, Kleeff J, Halloran CM, Raraty M, Jackson R, Melling J, et al. The Impact of Positive Resection Margins on Survival and Recurrence Following Resection and Adjuvant Chemotherapy for Pancreatic Ductal Adenocarcinoma. Ann Surg (2019) 269(3):520-9. doi: 10.1097/SLA.0000000000002557

5. Grossberg AJ, Chu LC, Deig CR, Fishman EK, Hwang WL, Maitra A, et al. Multidisciplinary Standards of Care and Recent Progress in Pancreatic Ductal Adenocarcinoma. CA Cancer J Clin (2020) 70(5):375-403. doi: 10.3322/caac.21626

6. Neoptolemos JP, Palmer DH, Ghaneh P, Psarelli EE, Valle JW, Halloran CM, et al. Comparison of Adjuvant Gemcitabine and Capecitabine With Gemcitabine Monotherapy in Patients With Resected Pancreatic Cancer (ESPAC-4): A Multicentre, Open-Label, Randomised, Phase 3 Trial. Lancet (2017) 389(10073):1011-24. doi: 10.1016/S0140-6736(16)32409-6

7. Conroy T, Hammel P, Hebbar M, Ben Abdelghani M, Wei AC, Raoul JL, et al. FOLFIRINOX or Gemcitabine as Adjuvant Therapy for Pancreatic Cancer. N Engl J Med (2018) 20379(25):2395-406. doi: 10.1056/NEJMoa1809775

8. Callery MP, Chang KJ, Fishman EK, Talamonti MS, William Traverso L, Linehan DC. Pretreatment Assessment of Resectable and Borderline Resectable Pancreatic Cancer: Expert Consensus Statement. Ann Surg Oncol (2009) 16(7):1727-33. doi: 10.1245/s10434-009-0408-6

9. Katz MHG, Marsh R, Herman JM, Shi Q, Collison E, Venook AP, et al. Borderline Resectable Pancreatic Cancer: Need for Standardization and Methods for Optimal Clinical Trial Design. Ann Surg Oncol (2013) 20 (8):2787-95. doi: 10.1245/s10434-013-2886-9

10. Bockhorn M, Uzunoglu FG, Adham M, Imrie C, Milicevic M, Sandberg AA, et al. Borderline Resectable Pancreatic Cancer: A Consensus Statement by the International Study Group of Pancreatic Surgery (ISGPS). Surg (2014) 155 (6):977-88. doi: 10.1016/j.surg.2014.02.001

11. Isaji S, Mizuno S, Windsor JA, Bassi C, Fernandez Del-Castilo C, Hackert T, et al. International Consensus on Definition and Criteria of Borderline

\section{DATA AVAILABILITY STATEMENT}

The raw data supporting the conclusions of this article will be made available by the authors, without undue reservation.

\section{AUTHOR CONTRIBUTIONS}

All authors have contributed equally to this manuscript in terms of data acquisition and analysis, and manuscript preparation and refinement.

\section{SUPPLEMENTARY MATERIAL}

The Supplementary Material for this article can be found online at: https://www.frontiersin.org/articles/10.3389/fonc.2021.791946/ full\#supplementary-material

Resectable Pancreatic Ductal Adenocarcinoma 2017. Pancreatol (2018) 18 (1):2-11. doi: 10.1016/j.pan.2017.11.011

12. Versteijne E, Suker M, Groothuis K, Akkermans-Vogelaar JM, Besselink MG, Bonsing BA, et al. Preoperative Chemoradiotherapy Versus Immediate Surgery for Resectable and Borderline Resectable Pancreatic Cancer: Results of the Dutch Randomized Phase III PREOPANC Trial. J Clin Oncol (2020) 38 (16):1763-73. doi: 10.1200/JCO.19.02274

13. Ghaneh P, Palmer DH, Cicconi S, Halloran C, Psarelli EE, Rawcliffe CL, et al. ESPAC-5f: Four-Arm, Prospective, Multicenter, International Randomized Phase II Trial of Immediate Surgery Compared With Neoadjuvant Gemcitabine Plus Capecitabine (GEMCAP) or FOLFIRINOX or Chemoradiotherapy (CRT) in Patients With Borderline Resectable Pancreatic Cancer. J Clin Oncol (2020) 38(15_suppl):4505-5. doi: 10.1200/ JCO.2020.38.15_suppl.4505

14. O’Reilly D, Fou L, Hasler E, Hawkins J, O'Connell S, Pelone F, et al. Diagnosis and Management of Pancreatic Cancer in Adults: A Summary of Guidelines From the UK National Institute for Health and Care Excellence. Pancreatol (2018) 18(8):962-70. doi: 10.1016/j.pan.2018.09.012

15. RICOCHET Study Group and West Midlands Research Collaborative. Receipt of Curative Resection or Palliative Care for Hepatopancreaticobiliary Tumours (RICOCHET): Protocol for a Nationwide Collaborative Observational Study. JMIR Res Protoc (2019) 8(7):e13566.

16. Harris PA, Taylor R, Thielke R, Payne J, Gonzalez N, Conde JG. Research Electronic Data Capture (REDCap)-A Metadata-Driven Methodology and Workflow Process for Providing Translational Research Informatics Support. J BioMed Inform (2009) 42(2):377-81. doi: 10.1016/j.jbi.2008.08.010

17. Eysenbach G. Improving the Quality of Web Surveys: The Checklist for Reporting Results of Internet E-Surveys (CHERRIES). J Med Internet Res (2004) 6(3):e34. doi: 10.2196/jmir.6.3.e34

18. Meguid RA, Ahuja N, Chang DC. What Constitutes a "High-Volume" Hospital for Pancreatic Resection? J Am Coll Surg (2008) 206(4):622.e1-9. doi: 10.1016/j.jamcollsurg.2007.11.011

19. Pancreatic Adenocarcinoma . NCCN Clinical Practice Guidelines in Oncology (NCCN Guidelines. Available at: https://www.nccn.org/.professionals/ physician_gls/pdf/pancreatic.pdf (Accessed December 17th, 2020).

20. Eisenhauer EA, Therasse P, Bogaerts J, Schwartz LH, Sargent D, Ford R, et al. New Response Evaluation Criteria in Solid Tumours: Revised RECIST Guideline (Version 1.1). Eur J Cancer (2009) 45(2):228-47. doi: 10.1016/ j.ejca.2008.10.026

21. Strobel O, Neoptolemos J, Jäger D, Buchler MW. Optimizing the Outcomes of Pancreatic Cancer Surgery. Nat Rev Clin Oncol (2019) 16(1):11-26. doi: 10.1038/s41571-018-0112-1 
22. Recommendations for Pancreatic Cancer in Adults: Diagnosis and Management NICE . Available at: https://www.nice.org.uk/guidance/ng85/ chapter/.Recommendations.

23. Müller PC, Hodson J, Kuemmerli C, Kalisvaart M, Pande R, Roberts KJ. Effect of Time to Surgery in Resectable Pancreatic Cancer: A Systematic Review and Meta-Analysis. Langenbecks Arch Surg (2020) 405(3):293-302. doi: 10.1007/ s00423-020-01893-0

24. Merkow RP, Bilimoria KY, Tomlinson JS, Paruch JL, Fleming JB, Talamonti MS, et al. Postoperative Complications Reduce Adjuvant Chemotherapy Use in Resectable Pancreatic Cancer. Ann Surg (2014) 260(2):372-7. doi: 10.1097/ SLA.0000000000000378

25. Wu W, He J, Cameron JL, Makary M, Soares K, Ahuja N, et al. The Impact of Postoperative Complications on the Administration of Adjuvant Therapy Following Pancreaticoduodenectomy for Adenocarcinoma. Ann Surg Oncol (2014) 21(9):2873-81. doi: 10.1245/s10434-014-3722-6

26. Strobel O, Lorenz P, Hinz U, Gaida M, Konig AK, Hank T, et al. Actual FiveYear Survival After Upfront Resection for Pancreatic Ductal Adenocarcinoma: Who Beats the Odds? Ann Surg (2020). doi: 10.1097/ SLA.0000000000004147

27. West HJ, Jin JO. JAMA Oncology Patient Page. Performance Status in Patients With Cancer. JAMA Oncol (2015) 1(7):998. doi: 10.1001/ jamaoncol.2015.3113

28. Fahrmann JF, Schmidt CM, Mao X, Irajizad E, Loftus M, Zhang J, et al. LeadTime Trajectory of CA19-9 as an Anchor Marker for Pancreatic Cancer Early Detection. Gastroenterology (2020) 160(4):1373-83.e6. doi: 10.1053/ j.gastro.2020.11.052

29. Groot VP, Gemenetzis G, Blair AB, Rivero-Soto J, Yu J, Javed AA, et al. Defining and Predicting Early Recurrence in 957 Patients With Resected Pancreatic Ductal Adenocarcinoma. Ann Surg (2019) 269(6):1154. doi: 10.1097/SLA.0000000000002734

30. Collisson EA, Bailey P, Chang DK, Biankin AV. Molecular Subtypes of Pancreatic Cancer. Nat Rev Gastroenterol Hepatol (2019) 16(4):207-20. doi: 10.1038/s41575-019-0109-y

31. Kim RY, Christians KK, Aldakkak M, Clarke CN, George B, Kamgar M, et al. Total Neoadjuvant Therapy for Operable Pancreatic Cancer. Ann Surg Oncol (2020) 28(4):2246-56. doi: 10.1245/s10434-020-09149-3

32. Ahmad SA, Duong M, Sohal DPS, Gandhi NS, Beg MS, Wang-Gillam A, et al. Surgical Outcome Results From SWOG S1505: A Randomized Clinical Trial of mFOLFIRINOX Versus Gemcitabine/Nab-Paclitaxel for Perioperative Treatment of Resectable Pancreatic Ductal Adenocarcinoma. Ann Surg (2020) 272(3):481-6. doi: 10.1097/SLA.0000000000004155

33. Strobel O, Hank T, Hinz U, Bergmann F, Schneider L, Springfield C, et al. Pancreatic Cancer Surgery: The New R-Status Counts. Ann Surg (2017) 265 (3):565-73. doi: 10.1097/SLA.0000000000001731

34. Hackert T, Sachsenmaier M, Hinz U, Schneider L, Michalski CW, Springfield L, et al. Locally Advanced Pancreatic Cancer: Neoadjuvant Therapy With Folfirinox Results in Resectability in 60\% of the Patients. Ann Surg (2016) 264 (3):457-63. doi: 10.1097/SLA.0000000000001850

35. Michelakos T, Pergolini I, Castillo CF-D, Honselmann KC, Cai L, Deshpande V, et al. Predictors of Resectability and Survival in Patients With Borderline and Locally Advanced Pancreatic Cancer Who Underwent Neoadjuvant Treatment With FOLFIRINOX. Ann Surg (2019) 269(4):733-40. doi: 10.1097/SLA.0000000000002600

36. Truty MJ, Kendrick ML, Nagorney DM, Smoot RL, Cleary SP, Graham RP, et al. Factors Predicting Response, Perioperative Outcomes, and Survival Following Total Neoadjuvant Therapy for Borderline/Locally Advanced Pancreatic Cancer. Ann Surg (2019) 273(2):341-9. doi: 10.1097/SLA.0000000000003284

37. Gemenetzis G, Groot VP, Blair AB, Laheru DA, Zheng L, Narang AK, et al. Survival in Locally Advanced Pancreatic Cancer After Neoadjuvant Therapy and Surgical Resection. Ann Surg (2019) 270(2):340-7. doi: 10.1097/SLA.0000000000002753

38. Attard JA, Farrugia A, Pathanki A, Roberts KJ, Dasari B, Isaac J, et al. Treatment Strategies for the Optimal Management of Locally Advanced Pancreatic Adenocarcinoma With Curative Intent: A Systematic Review. Pancreas (2020) 49(10):1264-75. doi: 10.1097/MPA.0000000000001694
39. Hammel P, Huguet F, van Laethem J-L, Goldstein D, Glimelius B, Artru P, et al. Effect of Chemoradiotherapy vs Chemotherapy on Survival in Patients With Locally Advanced Pancreatic Cancer Controlled After 4 Months of Gemcitabine With or Without Erlotinib: The LAP07 Randomized Clinical Trial. JAMA (2016) 315(17):1844-53. doi: 10.1001/jama.2016.4324

40. Katz MHG, Ou F-S, Herman JM, Ahmad SA, Wolpin B, Marsh R, et al. Alliance for Clinical Trials in Oncology (ALLIANCE) Trial A021501: Preoperative Extended Chemotherapy vs. Chemotherapy Plus Hypofractionated Radiation Therapy for Borderline Resectable Adenocarcinoma of the Head of the Pancreas. BMC Cancer (2017) 17 (1):505. doi: 10.1186/s12885-017-3441-z

41. Ferrone CR, Marchegiani G, Hong TS, Ryan DP, Deshpande V, McDonnell EI, et al. Radiological and Surgical Implications of Neoadjuvant Treatment With FOLFIRINOX for Locally Advanced and Borderline Resectable Pancreatic Cancer. Ann Surg (2015) 261(1):12-7. doi: 10.1097/ SLA.0000000000000867

42. Lee W, Oh M, Kim JS, Park Y, Kwon JW, Jun E, et al. Metabolic Activity by FDG-PET/CT After Neoadjuvant Chemotherapy in Borderline Resectable and Locally Advanced Pancreatic Cancer and Association With Survival. $\mathrm{Br} J$ Surg (2021), znab229. doi: 10.1093/bjs/znab229

43. Loos M, Kester T, Klaiber U, Mihalijevic AL, Mehrabi A, Muller-Stich BM, et al. Arterial Resection in Pancreatic Cancer Surgery: Effective After a Learning Curve. Ann Surg (2020). doi: 10.1097/SLA.0000000000004054

44. Małczak P, Sierżęga M, Stefura T, Dros J, Skomarovska O, et al. Arterial Resections in Pancreatic Cancer - Systematic Review and Meta-Analysis. HPB (2020) 22(7):961-8. doi: 10.1016/j.hpb.2020.04.005

45. Pawlik TM, Laheru D, Hruban RH, Coleman J, Wolfgang CL, Campbell K, et al. Evaluating the Impact of a Single-Day Multidisciplinary Clinic on the Management of Pancreatic Cancer. Ann Surg Oncol (2008) 15(8):2081-8. doi: 10.1245/s10434-008-9929-7

46. Brauer DG, Strand MS, Sanford DE, Kushnir VM, Lim KH, Mullady DK, et al. Utility of a Multidisciplinary Tumor Board in the Management of Pancreatic and Upper Gastrointestinal Diseases: An Observational Study. HPB (2017) 19 (2):133-9. doi: 10.1016/j.hpb.2016.11.002

47. Kirkegård J, Aahlin EK, Al-Saiddi M, Bratlie SO, Coolsen M, de Haas RJ, et al. Multicentre Study of Multidisciplinary Team Assessment of Pancreatic Cancer Resectability and Treatment Allocation. Br J Surg (2019) 106 (6):756-64. doi: 10.1002/bjs.11093

48. Jones CM, Radhakrishna G, Aitken K, Bridgewater J, Corrie P, Eatock M, et al. Considerations for the Treatment of Pancreatic Cancer During the COVID-19 Pandemic: The UK Consensus Position. Br J Cancer (2020) 123(5):709-13. doi: 10.1038/s41416-020-0980-x

49. Oba A, Stoop TF, Löhr M, Hackert T, Zyromski N, Nealon WH, et al. Global Survey on Pancreatic Surgery During the COVID-19 Pandemic. Ann Surg (2020) 272(2):e87. doi: 10.1097/SLA.0000000000004006

Conflict of Interest: The authors declare that the research was conducted in the absence of any commercial or financial relationships that could be construed as a potential conflict of interest.

Publisher's Note: All claims expressed in this article are solely those of the authors and do not necessarily represent those of their affiliated organizations, or those of the publisher, the editors and the reviewers. Any product that may be evaluated in this article, or claim that may be made by its manufacturer, is not guaranteed or endorsed by the publisher.

Copyright (c) 2021 Gemenetzis, McKay, Pathak, Moir, Laing, Jamieson, Young, Chatzizacharias, Giovinazzo and Roberts. This is an open-access article distributed under the terms of the Creative Commons Attribution License (CC BY). The use, distribution or reproduction in other forums is permitted, provided the original author(s) and the copyright owner(s) are credited and that the original publication in this journal is cited, in accordance with accepted academic practice. No use, distribution or reproduction is permitted which does not comply with these terms. 